Provided for non-commercial research and education use. Not for reproduction, distribution or commercial use.

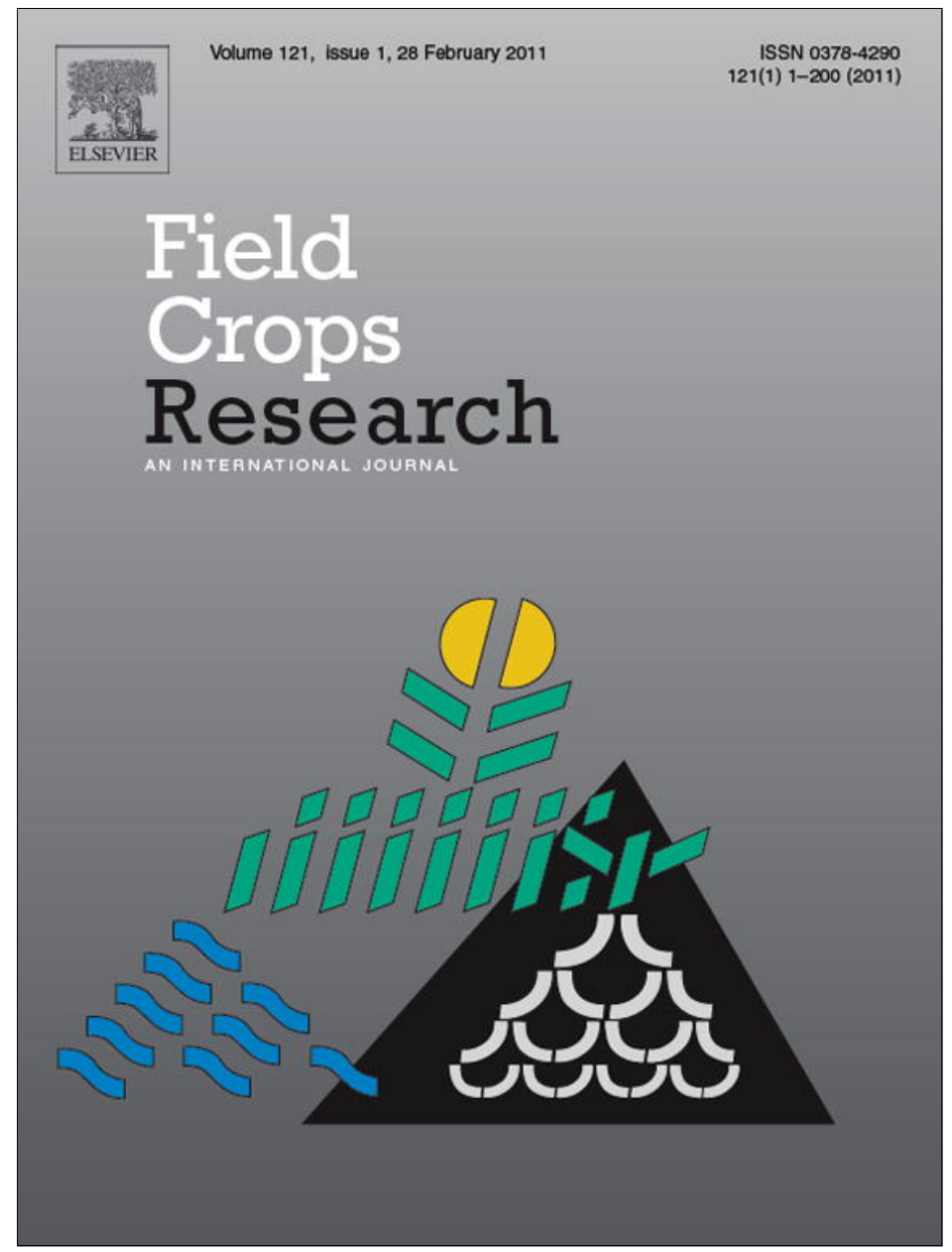

This article appeared in a journal published by Elsevier. The attached copy is furnished to the author for internal non-commercial research and education use, including for instruction at the authors institution and sharing with colleagues.

Other uses, including reproduction and distribution, or selling or licensing copies, or posting to personal, institutional or third party websites are prohibited.

In most cases authors are permitted to post their version of the article (e.g. in Word or Tex form) to their personal website or institutional repository. Authors requiring further information regarding Elsevier's archiving and manuscript policies are encouraged to visit:

http://www.elsevier.com/copyright 


\title{
Genetic diversity for grain nutrients contents in a core collection of finger millet (Eleusine coracana (L.) Gaertn.) germplasm
}

\author{
H.D. Upadhyaya ${ }^{\mathrm{a}, *}$, S. Ramesh ${ }^{\mathrm{b}}$, Shivali Sharma ${ }^{\mathrm{a}}$, S.K. Singh ${ }^{\mathrm{c}}$, S.K. Varshney ${ }^{\mathrm{c}}$, N.D.R.K. Sarma ${ }^{\mathrm{d}}$, \\ C.R. Ravishankar ${ }^{\mathrm{e}}$, Y. Narasimhudu ${ }^{\mathrm{f}}$, V.G. Reddy ${ }^{\mathrm{a}}$, K.L. Sahrawat ${ }^{\mathrm{a}}$, T.N. Dhanalakshmi ${ }^{\mathrm{b}}$, M.A. Mgonja ${ }^{\mathrm{g}}$, \\ H.K. Parzies ${ }^{\text {h }}$, C.L.L. Gowda ${ }^{a}$, Sube Singh ${ }^{\text {a }}$
}

a International Crops Research Institute for the Semi Arid Tropics (ICRISAT), Patancheru, Andhra Pradesh (AP), India

${ }^{\mathrm{b}}$ University of Agricultural Sciences (UAS), College of Agriculture, Bengaluru, Karnataka, India

${ }^{\mathrm{c}}$ Rajendra Agricultural University (RAU), Dholi-843121, Bihar, India

d Acharya NG Ranga Agricultural University (ANGRAU), Regional Agriculture Research Station (RARS), Vizianagaram-535001, AP, India

e UAS, Zonal Agricultural Research Station, Mandya-571405, Karnataka, India

${ }^{\mathrm{f}}$ Acharya NG Ranga Agricultural University (ANGRAU), Regional Agriculture Research Station (RARS), Nandyal-518502, AP, India

${ }^{g}$ International Crops Research Institute for the Semi Arid Tropics (ICRISAT)-Eastern and Southern Africa (ESA), PO Box 39063 Nairobi, Kenya

h University of Hohenheim, Institute of Plant Breeding, Seed Science and Population Genetics, Fruwirthstr. 21, D-70599, Stuttgart, Germany

\section{A R T I C L E I N F O}

\section{Article history:}

Received 10 September 2010

Received in revised form 8 November 2010

Accepted 16 November 2010

\section{Key words:}

Finger millet

Micronutrient contents of grain

Protein

Genetic diversity

\begin{abstract}
A B S T R A C T
Finger millet is a promising source of micronutrients and protein besides energy and can contribute to the alleviation of iron ( $\mathrm{Fe}$ ), zinc $(\mathrm{Zn})$ and protein malnutrition affecting women and preschool children in African and south-east Asian countries. The most cost effective approach for mitigating micronutrient and protein malnutrition is to introduce staple crop cultivars selected and/or bred for $\mathrm{Fe}, \mathrm{Zn}$ and protein dense grain. Breeding finger millet for enhanced grain nutrients is still in its infancy. Analysis, detection and exploitation of the existing variability among the germplasm accessions are the initial steps in breeding micronutrient and protein-dense finger millet cultivars. Evaluation of finger millet core collection for grain nutrients and agronomic traits revealed a substantial genetic variability for grain Fe, Zn, calcium (Ca) and protein contents. The accessions rich in nutrient contents were identified and their agronomic diversity assessed. The accessions rich in $\mathrm{Zn}$ content have significantly higher grain yield potential than those rich in Fe and protein content. Grain nutrient-specific accessions and those contrasting for nutrient contents were identified for use in the strategic research and cultivar development in finger millet.

() 2010 Elsevier B.V. All rights reserved.
\end{abstract}

\section{Introduction}

Finger millet (Eleusine coracana L.Gaertn.) ranks fourth in importance among millets in the world after sorghum (Sorghum bicolor), pearl millet (Pennisetum glaucum) and foxtail millet (Setaria italica) (Upadhyaya et al., 2007a). The crop was domesticated around 5000 years BC (Hilu and de Wet, 1976a). It is an allopolyploid with chromosome number $2 n=4 x=36$ and evolved from a cross between two diploid species, E. indica (AA) and E. floccifolia or E. tristachya (BB) as genome donors (Chennaveeraiah and Hiremath, 1973, 1974; Hilu and de Wet, 1976b; Hiremath and Salimath, 1992).

Finger millet is widely cultivated in Africa and South Asia under varied agro-climatic conditions (Dida et al., 2008). In Africa, it is extensively cultivated in Uganda, Kenya, Tanzania, Ethiopia,

\footnotetext{
* Corresponding author at: International Crops Research Institute for the Semi Arid Tropics (ICRISAT), Patancheru, Hyderabad, PO, 502324, AP, India. Tel.: +91 40 30713333; fax: +91 $4030713074 / 5$.

E-mail address: h.upadhyaya@cgiar.org (H.D. Upadhyaya).
}

Rwanda, Burundi, Zambia and Malawi (Mnyenyembe and Gupta, 1998; Obilana et al., 2002). In south Asia, finger millet is widely cultivated in India and Nepal (Upadhyaya et al., 2007b). The precise data on world area under finger millet are not available, because it is frequently reported with other millets including pearl millet (e.g., in the FAO database). However, as per the estimate by the Consultative Group on International Agricultural Research (CGIAR), finger millet contributes 10 per cent of the total area ( 34.6 million ha) planted to millets (FAO, 2004). In India, finger millet ranks next to pearl millet and is cultivated on $2.6 \mathrm{~m}$ ha area with a production of about $3.00 \mathrm{mt}$ (www.indiastat.com).

Finger millet is a nutritious food grain crop with a fair amount

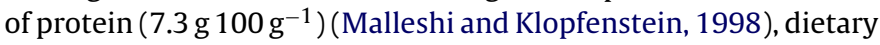
fibre (15-20\%) (Chethan and Malleshi, 2007) and a rich source of calcium (Ca) (344 mg $100 \mathrm{~g}^{-1}$ ) (Gopalan et al., 2002). Wider adaptability (Upadhyaya et al., 2007b), higher nutritional quality (Gopalan et al., 2002), higher multiplication rate and longer shelf life under ambient conditions (Iyengar et al., 1945), makes finger millet an ideal crop for use as a staple food and for famine reserve. 
Finger millet being a promising source of micronutrients and protein (Malleshi and Klopfenstein, 1998) besides energy, can make a contribution to alleviating micronutrient and protein malnutrition, also called 'hidden-hunger', affecting more than half of the world's population, especially women and preschool children in most countries of Africa and south-east Asia (Underwood, 2000). Malnutrition due to protein deficiency is also alarming in the Indian subcontinent (Chand et al., 2003; Prasad, 2010). Intake of diet poor in iron $(\mathrm{Fe})$, zinc $(\mathrm{Zn})$ and protein is the major cause for micronutrient and protein malnutrition. Fe deficiency leads to anaemia; about $79 \%$ of the pre-school children between 6 and 35 months of age and $56 \%$ of women between 15 and 49 years of age are anaemic in India (Krishnaswamy, 2009). Protein deficiency causes retarded physical and mental growth, while $\mathrm{Zn}$ deficiency leads to diarrhoea, pneumonia and reduced immunity to diseases, and in pregnant women it leads to infant mortality (Gibson et al., 2008). The high proportion of carbohydrates in form of non-starchy polysccharides and dietry fibres in grains helps in reducing cholesterol and slow release of glucose to the blood stream during digestion and hence suitable for the diabetic patients. Because of its high nutrient contents, finger millet is gaining importance in Europe and USA where it has potential for use in variety of foods such as porridge, bread, biscuits, pastas, instant baby food, and composite flour (Dendy, 1993; Senthil et al., 2005).

The most cost effective approach for mitigating micronutrient and protein malnutrition is to introduce finger millet varieties selected and/or bred for increased $\mathrm{Fe}, \mathrm{Zn}$ and protein contents through plant breeding. Plant breeding approach scores over others (such as food fortification, micronutrient supplements, dietary strategies and medical interventions) because it (i) complements the existing approaches to combat micronutrient deficiency, (ii) does not require any special program to change the behaviour of farmers/consumers, and (iii) cultivars rich in Fe, $\mathrm{Zn}$ and protein with farmer preferred grain quality and adaptation traits are readily accepted (Welch and Graham, 2004; Graham et al., 2007; Pfeiffer and McClafferty, 2007; Prasad, 2010).

Attempts to breed finger millet for enhanced grain micronutrient and protein contents are still in its infancy. Exploitation of existing variability among germplasm accessions is the first step and short-term strategy for developing and delivering micronutrient and protein-dense finger millet cultivars to address the micronutrient and protein malnutrition in the target population. The core collection (Upadhyaya et al., 2006) which captures most of the variability of the entire finger millet collection of 5940 accessions developed and maintained at the International Crops Research Institute of Semi-Arid Tropics (ICRISAT), Patancheru, Andhra Pradesh, India is the gateway for effective utilization of genetic resources for developing grain nutrient-rich finger millet cultivars. The objective of the present investigation is to assess genetic variability and identify promising accessions for grain $\mathrm{Fe}$, $\mathrm{Zn}$, Ca and protein contents and agronomic traits in a core collection of finger millet germplasm for use in the crop improvement programmes.

\section{Material and methods}

\subsection{Material}

The material for the study consisted of 622 core accessions (Upadhyaya et al., 2006), four common control cultivars, PR 202, RAU 8, VR 149 and VR 708 and two location-specific controls. The control cultivars are the high yielding cultivars developed and released for commercial cultivation in India with a grain yield potential of 2.5-3.5 $\mathrm{tha}^{-1}$ (Seetharam et al., 2006). PR 202 [medium duration and dual-purpose (grain + stover cultivar)] and
VR 708 (short duration and blast tolerant) are pure-line selections from landrace cultivars, Peddapuram local and VMEC-32, respectively released for commercial cultivation in Andhra Pradesh state (Seetharam et al., 2006). RAU 8 is a medium duration, grain-purpose and blast tolerant cultivar developed by pedigree selection from the segregating population derived from BR $407 \times$ Ranchi Local cross for commercial cultivation in Bihar state (Seetharam et al., 2006). VL 149 is a short duration, cold tolerant grain-purpose cultivar developed by pedigree selection from VL $201 \times$ IE 882 cross for commercial cultivation in high altitudes of Almora in Uttarakhand state (Seetharam et al., 2006). This cultivar (VL 149) is being used as a National Check in the field trials for finger millet improvement coordinated by the All India Coordinated Small Millet Improvement Project Cell located in Bangalore, India (Seetharam et al., 2006).

\subsection{Field evaluation}

The 622 core collection accessions, four common control cultivars, and two location-specific controls were evaluated at five locations in India during the 2008 rainy season. The test locations included; ICRISAT, Patancheru $\left(17.3^{\circ} \mathrm{N} 78.5^{\circ} \mathrm{E}\right)$, Vizianagaram $\left(18.7^{\circ} \mathrm{N} 83.3^{\circ} \mathrm{E}\right)$ and Nandhyal $\left(15.3^{\circ} \mathrm{N} 78.3^{\circ} \mathrm{E}\right)$ in Andhra Pradesh, Mandya $\left(12.3^{\circ} \mathrm{N} 76.5^{\circ} \mathrm{E}\right)$ in Karnataka, and Dholi $\left(24.9^{\circ} \mathrm{N} 72.1^{\circ} \mathrm{E}\right)$ in Bihar in India. The accessions were planted in alpha design (Paterson and Williams, 1976) with two replications at Patancheru. At other locations, the experiment was conducted in augmented design (Federer, 1961) with one of the six controls repeated after every nine test entries. Each accession was grown in a single row of $4 \mathrm{~m}$ length by maintaining a row-to-row spacing of $0.6 \mathrm{~m}$ at Patancheru, $0.4 \mathrm{~m}$ at Dholi, $0.3 \mathrm{~m}$ at Mandya, Nandhyal and Vizianagaram. The plant-to-plant spacing within a row was fixed at $0.1 \mathrm{~m}$ at all the locations. Basal fertilizer dose of $20 \mathrm{~kg} \mathrm{~N}$ and $50 \mathrm{~kg} \mathrm{P}$ and a top dressing of $50 \mathrm{~kg} \mathrm{Nha}^{-1}$ were applied 30 days after sowing (DAS) at all locations. The experimental plots were maintained free of weeds and insect-pests. Protective irrigation was provided whenever necessary.

\subsection{Grain nutrient determination}

The nutrient content in grains was determined only for the core collection accessions evaluated at the ICRISAT Center, Patancheru. Care was taken to avoid the contamination of grains with dust and metal particles during their cleaning. The grain samples from each replication were collected in clean cloth bags and sent to the Central Analytical Services laboratory in ICRISAT, Patancheru, India for the estimation of $\mathrm{Fe}, \mathrm{Zn}, \mathrm{Ca}$ and protein content (\%). The grain samples of core accessions from two replications were powdered and digested using the tri-acid mixture, and $\mathrm{Fe}, \mathrm{Zn}$ and $\mathrm{Ca}$ contents in the digests were determined by atomic absorption spectrophotometer (Sahrawat et al., 2002). Protein content in grain samples was determined in the digests using an Autoanalyser (Singh and Jambunathan, 1980). Beta-carotene content was analyzed in seed samples using high performance liquid chromatography according to Weissenberg et al. (1997)

\subsection{Recording of data on quantitative and qualitative traits}

Five representative plants were labelled in each plot for recording data on 15 quantitative traits (days to flowering, plant height, basal tillers, flag leaf blade length and width, flag leaf sheath length, peduncle length, ear head exertion, ear head length and width, length and width of longest finger, fingers per ear head, grain yield and overall plant aspect score) following finger millet descriptors (IBPGR, 1985). Data on 5 qualitative (plant pigmentation, growth habit, inflorescence (ear head) compactness and shape, culm branching and grain colour) were recorded on plot 
basis. Data on plant pigmentation and growth habit were recorded after $50 \%$ flowering. Grain characteristics were recorded at postharvest stage in the laboratory. The number of days to flowering was recorded as the number of days from sowing to the date when $50 \%$ plants in a plot started flowering. Data on plant height, basal tillers, flag leaf blade length and width, flag leaf sheath length, peduncle length, ear head exertion, ear head length and width, length and width of longest finger, fingers per ear head, and grain yield per plant were recorded on five representative plants. Grain yield of 5 plants was added to the plot yield to determine total plot yield in $\mathrm{kg} \mathrm{ha}^{-1}$. Ear head exertion was measured as length of the exposed peduncle from the flag leaf to the base of the ear. Ear head length and width were measured at maturity as the maximum length from the base to the tip of the ear head, and maximum width in natural position. For quantitative traits, the averages of five plants per plot were computed and used for statistical analysis of the data.

\subsection{Data analysis}

\subsubsection{Grain nutrients contents and quantitative traits}

The replicated mean values of the four grain nutrient contents of the 622 core accessions and four control cultivars were used to estimate first-degree statistics such as mean, and second-degree statistics such as phenotypic variance $\left(\sigma_{\mathrm{p}}^{2}\right)$ and genotypic variance $\left(\sigma_{\mathrm{g}}^{2}\right)$ following the Restricted Maximum Likelihood (REML) method and random model considering accessions as random effects (Hardy and Thompson, 1996; Payne and Senn, 2007) using the GenStat software, version 10 (http://www.genstat.co.uk). Best Linear Unbiased Predictors (BLUPs) of each of grain nutrient and accession were estimated (Schonfeld and Werner, 1986). As grain nutrients are measured in different units, estimates of $\sigma_{\mathrm{p}}^{2}$ and $\sigma_{\mathrm{g}}^{2}$ are not comparable across grain nutrients. Therefore, $\sigma_{\mathrm{p}}^{2}$ and $\sigma_{\mathrm{g}}^{2}$ were standardized into unit-free estimates as; phenotypic coefficient of variability $(\mathrm{PCV})=\left[\left(\sigma_{p} / \bar{u}\right) \times 100\right]$ and genotypic coefficient of variability $(\mathrm{GCV})=\left[\left(\sigma_{g} / \bar{u}\right) \times 100\right]$, where, $\bar{u}$ is the trial mean of grain nutrients. Broad-sense heritability of all the grain nutrients were estimated as the ratio of $\sigma_{\mathrm{g}}^{2}$ to $\sigma_{\mathrm{p}}^{2}$ and expressed in percentage.

Location-wise and pooled analyses of combined data on quantitative traits from all the locations were carried out to dissect total variability of the entries $\left(\sigma_{\mathrm{p}}^{2}\right)$ into sources attributable to genotype $\left(\sigma_{\mathrm{g}}^{2}\right)$, location $\left(\sigma_{1}^{2}\right)$, their interaction $\left(\sigma_{\mathrm{g} \times 1}^{2}\right)$ and un-controlled experimental error $\left(\sigma_{\mathrm{e}}^{2}\right)$ following REML method and mixed model considering accessions as random effects and locations as fixed effects (Hardy and Thompson, 1996; Payne and Senn, 2007) using the GenStat software, version 10 (http://www.genstat.co.uk). BLUPs of each quantitative trait and accession were estimated for individual location and pooled data (Schonfeld and Werner, 1986). Significance of variability due to location environmental effects was tested using Wald (1943) statistic.

For each of the nutrients, the best 15 accessions were selected and their agronomic diversity (for selected traits) was assessed separately using descriptive statistics such as mean, range and variance. The differences in the means of the selected quantitative traits between the best accessions (common accessions were excluded from analysis) for each of the nutrients were tested for their statistical significance using two-sample ' $t$ ' statistic, while the variances were tested using ' $F$ ' statistic (Snedecor and Cochran, 1994).

Considering the mean of the control cultivars as the bench mark, a total of 24 accessions ( 20 accessions rich in all the four nutrients, 2 rich in Fe, $\mathrm{Zn}$ and protein contents and 2 rich in $\mathrm{Ca}$ and protein contents) were selected and their diversity for agronomic traits assessed. Principal component analysis (PCA) (Pearson, 1901) was carried out using adjusted, standardized and uncorrelated data of selected quantitative traits of these 24 accessions to reduce the dimensionality of the data into principal components (PCs) and a biplot was generated using the GenStat software. Based on the first three PCs which explained 75\% variability, the 24 accessions were grouped into four clusters following Ward's (1963) agglomerative hierarchical clustering algorithm. The mean and variance of all the quantitative traits of the accessions included in each cluster were estimated. The differences in the cluster means of each quantitative trait were tested for their statistical significance using Newman (1939)-Keuls (1952) procedure. The homogeneity of variances of each quantitative trait across the clusters was tested for their statistical significance using Levene's (1960) test.

\subsubsection{Interrelationships among grain nutrients contents and with agronomic traits}

Correlation coefficients (Snedecor and Cochran, 1994) among the four grain nutrients and between nutrients and agronomic traits were estimated to examine association among the nutrients and their association with agronomic traits.

\subsubsection{Analysis of association between grain nutrient contents with qualitative traits and geographical origin}

The accessions were classified into three categories as decumbent, erect and prostrate based on the growth habit, into two categories of purple and green based on plant pigmentation, into four races such as plana, elongate, compacta, and vulgaris based on ear head compactness and shape and five categories as dark brown, light brown, ragi brown, reddish brown and white based on grain colour. Based on geographical origin, the accessions were classified into four categories as those originating from Africa, Asia, Americas and Europe. Those with unknown origin were categorised into a separate group. The differences between the means of four grain nutrient contents of core accessions classified based on growth habit, plant pigmentation, ear head compactness and shape and grain colour and geographical origin, were tested for their statistical significance using Newman (1939)-Keuls (1952) procedure to determine the relationship of grain nutrient contents with qualitative trait groups and geographical origin.

\section{Results}

Substantial variability for all the four grain nutrients was evident from the estimates of range $\left(21.71-65.23 \mathrm{mg} \mathrm{kg}^{-1}\right.$ for $\mathrm{Fe}$; $16.58-25.33 \mathrm{mg} \mathrm{kg}^{-1}$ for $\mathrm{Zn}$; $1.84-4.89 \mathrm{~g} \mathrm{~kg}^{-1}$ for Ca; $6.00-11.09 \%$ for protein), and PCV and GCV (22.87 and $17.87 \%$ for Fe; 12.69 and $8.85 \%$ for $\mathrm{Zn} ; 18.80$ and $17.75 \%$ for Ca; 10.54 and $8.79 \%$ for protein). A comparative analysis revealed that the mean grain nutrient contents of the core accessions (with $29.32 \mathrm{mg} \mathrm{kg}^{-1} \mathrm{Fe}, 19.91 \mathrm{mg} \mathrm{kg}^{-1}$ $\mathrm{Zn} ; 2.85 \mathrm{~g} \mathrm{~kg}^{-1} \mathrm{Ca}$ and $7.32 \%$ protein) were comparable to those of the control cultivars $\left(31.05 \mathrm{mg} \mathrm{kg}^{-1} \mathrm{Fe}, 19.89 \mathrm{mg} \mathrm{kg}^{-1} \mathrm{Zn}\right.$; $2.87 \mathrm{~g} \mathrm{~kg}^{-1} \mathrm{Ca}$ and $7.30 \%$ protein).

The accessions such as IE $4708\left(65.23 \mathrm{mg} \mathrm{kg}^{-1}\right)$, IE 2921 (59.09 $\mathrm{mg} \mathrm{kg}^{-1}$ ), IE 4709 (48.56 $\mathrm{mg} \mathrm{kg}^{-1}$ ), IE 588 (45.91 $\mathrm{mg} \mathrm{kg}^{-1}$ ), IE $5736\left(45.55 \mathrm{mg} \mathrm{kg}^{-1}\right)$ and IE $4476\left(44.94 \mathrm{mg} \mathrm{kg}^{-1}\right)$ had higher $\mathrm{Fe}$ content based on the least significant difference (LSD) (Table 1). Similarly, the accessions, IE $3120\left(25.33 \mathrm{mg} \mathrm{kg}^{-1}\right)$, IE 7508 (24.16 $\left.\mathrm{mg} \mathrm{kg}^{-1}\right)$, IE $6546\left(23.94 \mathrm{mg} \mathrm{kg}^{-1}\right)$ and IE 3025 (23.63 $\left.\mathrm{mg} \mathrm{kg}^{-1}\right)$ and IE $7386\left(23.48 \mathrm{mg} \mathrm{kg}^{-1}\right)$ were superior in grain Zn (Table 2). IE $4476\left(4.89 \mathrm{~g} \mathrm{~kg}^{-1}\right)$, IE $2030\left(4.69 \mathrm{~g} \mathrm{~kg}^{-1}\right)$, IE 6546 (4.61 $\left.\mathrm{g} \mathrm{kg}^{-1}\right)$, IE $4708\left(4.51 \mathrm{~g} \mathrm{~kg}^{-1}\right)$, IE $2568\left(4.51 \mathrm{~g} \mathrm{~kg}^{-1}\right)$, IE 2957 $\left(4.47 \mathrm{~g} \mathrm{~kg}^{-1}\right)$ and IE $6537\left(4.39 \mathrm{~g} \mathrm{~kg}^{-1}\right)$ are some of the accessions which had significantly higher Ca content when compared to trial mean plus even two units of LSD (Table 3). Similarly, the accessions such as IE 6537 (11.09\%), IE 0009 (10.44\%), IE 4709 (9.95\%), IE 4708 (9.51\%), IE 6541 (9.46\%), IE 2921 (9.35\%), IE 6546 (9.30\%) and IE $4476(9.01 \%)$ had higher protein content when compared to trial mean plus two units of LSD (Table 4). 
Table 1

Performance of 15 best Fe-rich finger millet core collection accessions for selected agronomic traits.

\begin{tabular}{|c|c|c|c|c|c|c|c|c|c|}
\hline Accession no. & Country & $\begin{array}{l}\mathrm{Fe} \\
\left(\mathrm{mg} \mathrm{kg}^{-1}\right)\end{array}$ & $\begin{array}{l}\text { Days to } 50 \% \\
\text { flowering }\end{array}$ & $\begin{array}{l}\text { Plant } \\
\text { height }(\mathrm{cm})\end{array}$ & Basal tillers & $\begin{array}{l}\text { Ear head } \\
\text { length } \\
(\mathrm{mm})\end{array}$ & $\begin{array}{l}\text { Ear head } \\
\text { width } \\
(\mathrm{mm})\end{array}$ & $\begin{array}{l}\text { Fingers per } \\
\text { ear head }\end{array}$ & $\begin{array}{l}\text { Grain yield } \\
\left(\mathrm{kg} \mathrm{ha}^{-1}\right)\end{array}$ \\
\hline IE 4708 & Burundi & 65.23 & 62.4 & 109.8 & 6.5 & 104.2 & 49.0 & 8.8 & 553.5 \\
\hline IE 2921 & Malawi & 59.09 & 78.4 & 120.8 & 6.6 & 122.9 & 56.1 & 9.0 & 631.5 \\
\hline IE 4709 & Burundi & 48.56 & 64.2 & 113.9 & 8.1 & 124.7 & 47.4 & 8.3 & 464.0 \\
\hline IE 588 & India & 45.91 & 51.4 & 81.5 & 5.1 & 53.9 & 51.3 & 7.4 & 1058.7 \\
\hline IE 5736 & Nepal & 45.55 & 56.1 & 84.2 & 4.3 & 66.5 & 51.1 & 6.6 & 1019.6 \\
\hline IE 4476 & Zimbabwe & 44.94 & 72.1 & 91.7 & 4.6 & 104.5 & 52.5 & 8.3 & 543.2 \\
\hline IE 942 & India & 40.60 & 62.9 & 96.1 & 4.1 & 102.7 & 52.2 & 7.9 & 1293.7 \\
\hline IE 4734 & India & 39.63 & 53.5 & 82.2 & 4.4 & 56.2 & 53.1 & 6.1 & 1170.1 \\
\hline IE 5794 & Nepal & 39.11 & 68.4 & 96.4 & 4.6 & 66.5 & 51.7 & 7.6 & 1684.7 \\
\hline IE 4107 & Uganda & 39.02 & 70.4 & 102.5 & 4.1 & 101.1 & 51.7 & 7.6 & 1243.3 \\
\hline IE 7338 & Kenya & 38.90 & 78.8 & 106.9 & 4.2 & 97.1 & 51.7 & 6.3 & 1156.8 \\
\hline IE 2093 & India & 38.17 & 56.1 & 80.2 & 4.8 & 57.6 & 50.5 & 6.2 & 1175.1 \\
\hline IE 5870 & Nepal & 38.05 & 64.0 & 85.9 & 3.7 & 62.9 & 52.0 & 8.7 & 740.3 \\
\hline IE 4443 & Cameroon & 37.90 & 70.0 & 87.9 & 4.6 & 62.7 & 52.4 & 7.3 & 2317.3 \\
\hline IE 817 & India & 37.66 & 66.9 & 100.1 & 5.3 & 78.9 & 50.2 & 7.2 & 1239.7 \\
\hline Mean & & 43.89 & 65.0 & 96.0 & 5.0 & 84.1 & 51.5 & 7.5 & 1086.1 \\
\hline $\mathrm{SE} \pm$ & & 2.14 & 2.16 & 3.30 & 0.3 & 6.45 & 0.50 & 0.25 & 125.02 \\
\hline Least significant difference (LSD) & & 10.89 & & & & & & & \\
\hline Variance & & 68.44 & 69.76 & 162.95 & 1.39 & 623.35 & 3.74 & 0.91 & 234451.44 \\
\hline Range & & 27.57 & 27.4 & 40.6 & 4.4 & 70.9 & 8.7 & 2.9 & 1853.3 \\
\hline Minimum & & 37.66 & 51.4 & 80.2 & 3.7 & 53.9 & 47.4 & 6.1 & 464.0 \\
\hline Maximum & & 65.23 & 78.8 & 120.8 & 8.1 & 124.7 & 56.1 & 9.0 & 2317.3 \\
\hline \multicolumn{10}{|c|}{ Controlcultivars } \\
\hline IE 2043 (PR 202) & India & 26.51 & 71.4 & 98.3 & 5.1 & 76.4 & 54.0 & 7.7 & 2633.3 \\
\hline IE 3618 (RAU 8) & India & 31.49 & 65.4 & 95.0 & 5.0 & 78.1 & 52.1 & 7.7 & 2264.5 \\
\hline IE 4673 (VL 149) & India & 35.99 & 65.3 & 97.4 & 4.8 & 89.8 & 53.3 & 8.4 & 2002.6 \\
\hline VR 708 & India & 30.19 & 54.4 & 87.1 & 4.5 & 65.1 & 50.3 & 7.9 & 1553.3 \\
\hline Mean of control cultivars & & 31.05 & 64.1 & 94.5 & 4.9 & 77.4 & 52.5 & 7.9 & 2113.5 \\
\hline
\end{tabular}

The accessions, IE 4708 and IE 4476 were significantly superior in grain $\mathrm{Fe}, \mathrm{Ca}$ and protein, while IE 6537 was superior in grain $\mathrm{Ca}$ and protein contents based on trial mean +1 LSD.

The differences between the PCV and GCV estimates were relatively narrow for $\mathrm{Ca}(1.05)$ and protein (1.75) contents and large for Fe (5.05) and $\mathrm{Zn}$ (3.84) contents. The broad-sense heritability estimates were relatively higher for $\mathrm{Ca}(89.3 \%)$ and protein $(69.6 \%)$ and lower for $\mathrm{Zn} \mathrm{(48.6 \% )} \mathrm{and} \mathrm{Fe}(60.7 \%)$ contents.

Beta-carotene contents of seed samples showed values around the detection limit $\left(1 \mu \mathrm{g} \quad 100 \mathrm{~g}^{-1}\right.$ seeds $)$ and no considerable variation for the trait could be detected.

Table 2

Performance of 15 best $\mathrm{Zn}$-rich finger millet core collection accessions for selected agronomic traits.

\begin{tabular}{|c|c|c|c|c|c|c|c|c|c|}
\hline Accession no. & Country & $\begin{array}{l}\mathrm{Zn} \\
\left(\mathrm{mg} \mathrm{kg}^{-1}\right)\end{array}$ & $\begin{array}{l}\text { Days to } 50 \% \\
\text { flowering }\end{array}$ & $\begin{array}{l}\text { Plant } \\
\text { height }(\mathrm{cm})\end{array}$ & Basal tillers & $\begin{array}{l}\text { Ear head } \\
\text { length } \\
(\mathrm{mm})\end{array}$ & $\begin{array}{l}\text { Ear head } \\
\text { width } \\
(\mathrm{mm})\end{array}$ & $\begin{array}{l}\text { Fingers per } \\
\text { ear head }\end{array}$ & $\begin{array}{l}\text { Grain yield } \\
\left(\mathrm{kg} \mathrm{ha}^{-1}\right)\end{array}$ \\
\hline IE 3120 & India & 25.33 & 62.9 & 91.6 & 3.9 & 71.5 & 53.9 & 7.8 & 1112.0 \\
\hline IE 7508 & Ethiopia & 24.16 & 66.9 & 101.9 & 4.0 & 79.6 & 51.7 & 8.1 & 1338.0 \\
\hline IE 6546 & Nigeria & 23.94 & 88.4 & 99.2 & 4.8 & 126.5 & 54.9 & 8.4 & 1464.6 \\
\hline IE 3025 & Ethiopia & 23.63 & 81.8 & 109.7 & 4.1 & 131.0 & 53.2 & 7.8 & 1061.1 \\
\hline IE 7386 & Kenya & 23.48 & 71.6 & 110.5 & 4.4 & 86.7 & 51.8 & 8.1 & 1354.8 \\
\hline IE 7407 & Kenya & 23.31 & 75.5 & 103.4 & 4.3 & 64.8 & 54.1 & 7.3 & 1468.7 \\
\hline IE 615 & India & 23.17 & 65.0 & 102.2 & 4.5 & 76.0 & 51.1 & 6.9 & 1977.5 \\
\hline IE 712 & India & 22.90 & 66.9 & 99.0 & 4.1 & 73.6 & 53.2 & 7.2 & 1797.5 \\
\hline IE 5788 & Nepal & 22.83 & 65.7 & 98.6 & 4.3 & 65.9 & 51.5 & 6.8 & 1758.5 \\
\hline IE 633 & India & 22.80 & 63.5 & 100.8 & 4.6 & 70.8 & 52.0 & 7.5 & 1650.4 \\
\hline IE 2008 & India & 22.78 & 67.8 & 98.6 & 4.8 & 69.6 & 51.5 & 6.6 & 2095.7 \\
\hline IE 1023 & Unknown & 22.66 & 73.6 & 97.5 & 3.7 & 69.5 & 53.4 & 7.5 & 1468.9 \\
\hline IE 886 & Pakistan & 22.53 & 67.2 & 99.2 & 5.0 & 72.4 & 52.1 & 7.1 & 1729.4 \\
\hline IE 4817 & India & 22.46 & 64.7 & 89.1 & 4.8 & 64.4 & 50.3 & 7.8 & 1173.8 \\
\hline IE 510 & India & 22.46 & 62.6 & 100.5 & 5.2 & 68.0 & 49.5 & 7.6 & 1813.1 \\
\hline Mean & & 23.23 & 69.6 & 100.1 & 4.4 & 79.4 & 52.3 & 7.5 & 1550.9 \\
\hline $\mathrm{SE} \pm$ & & 0.20 & 1.92 & 1.42 & 0.11 & 5.39 & 0.38 & 0.14 & 80.85 \\
\hline Least significant difference (LSD) & & 3.50 & & & & & & & \\
\hline Variance & & 0.62 & 55.22 & 30.40 & 0.19 & 436.12 & 2.19 & 0.28 & 98049.36 \\
\hline Range & & 2.87 & 25.8 & 21.3 & 1.5 & 66.6 & 5.4 & 1.9 & 1034.6 \\
\hline Minimum & & 22.46 & 62.6 & 89.1 & 3.7 & 64.4 & 49.5 & 6.6 & 1061.1 \\
\hline Maximum & & 25.33 & 88.4 & 110.5 & 5.2 & 131.0 & 54.9 & 8.4 & 2095.7 \\
\hline IE 2043 (PR 202) & India & 18.89 & 71.4 & 98.3 & 5.1 & 76.4 & 54.0 & 7.7 & 2633.3 \\
\hline IE 3618 (RAU 8) & India & 19.71 & 65.4 & 95.0 & 5.0 & 78.1 & 52.1 & 7.7 & 2264.5 \\
\hline IE 4673 (VL 149) & India & 21.39 & 65.3 & 97.4 & 4.8 & 89.8 & 53.3 & 8.4 & 2002.6 \\
\hline VR 708 & India & 19.57 & 54.4 & 87.1 & 4.5 & 65.1 & 50.3 & 7.9 & 1553.3 \\
\hline Mean of control cultivars & & 19.89 & 64.1 & 94.4 & 4.8 & 77.3 & 52.4 & 7.9 & 2113.5 \\
\hline
\end{tabular}


Table 3

Performance of 15 best Ca-rich finger millet core collection accessions for selected agronomic traits.

\begin{tabular}{|c|c|c|c|c|c|c|c|c|c|}
\hline Accession no. & Country & $\mathrm{Ca}\left(\mathrm{g} \mathrm{kg}^{-1}\right)$ & $\begin{array}{l}\text { Days to } 50 \% \\
\text { flowering }\end{array}$ & $\begin{array}{l}\text { Plant } \\
\text { height }(\mathrm{cm})\end{array}$ & Basal tillers & $\begin{array}{l}\text { Ear head } \\
\text { length } \\
(\mathrm{mm})\end{array}$ & $\begin{array}{l}\text { Ear head } \\
\text { width } \\
(\mathrm{mm})\end{array}$ & $\begin{array}{l}\text { Fingers per } \\
\text { ear head }\end{array}$ & $\begin{array}{l}\text { Grain yield } \\
\left(\mathrm{kg} \mathrm{ha}^{-1}\right)\end{array}$ \\
\hline IE 4476 & Zimbabwe & 4.89 & 72.1 & 91.7 & 4.6 & 104.5 & 52.5 & 8.3 & 543.2 \\
\hline IE 2030 & India & 4.69 & 64.8 & 94.0 & 5.0 & 70.6 & 53.4 & 7.4 & 1958.2 \\
\hline IE 6546 & Nigeria & 4.61 & 88.4 & 99.2 & 4.8 & 126.5 & 54.9 & 8.4 & 1464.6 \\
\hline IE 4708 & Burundi & 4.51 & 62.4 & 109.8 & 6.5 & 104.2 & 49.0 & 8.8 & 553.5 \\
\hline IE 2568 & Kenya & 4.51 & 96.4 & 101.9 & 4.1 & 103.8 & 52.4 & 6.8 & 1031.3 \\
\hline IE 2957 & Germany & 4.47 & 59.9 & 91.4 & 4.0 & 82.5 & 50.5 & 6.9 & 1262.6 \\
\hline IE 6537 & Nigeria & 4.39 & 86.6 & 90.8 & 4.1 & 98.8 & 53.7 & 7.6 & 762.6 \\
\hline IE 2608 & Malawi & 4.24 & 79.4 & 100.9 & 5.4 & 115.5 & 54.5 & 6.7 & 1990.9 \\
\hline IE 2572 & Kenya & 4.21 & 93.7 & 99.1 & 5.5 & 111.1 & 54.0 & 6.8 & 1506.4 \\
\hline IE 2921 & Malawi & 4.19 & 78.4 & 120.8 & 6.6 & 122.8 & 56.1 & 9.0 & 631.5 \\
\hline IE 4443 & Cameroon & 4.15 & 70.0 & 87.9 & 4.6 & 62.7 & 52.4 & 7.3 & 2317.3 \\
\hline IE 2780 & Malawi & 4.00 & 81.6 & 107.3 & 6.7 & 116.2 & 52.2 & 7.4 & 1534.7 \\
\hline IE 4866 & India & 3.93 & 82.6 & 112.8 & 4.4 & 87.8 & 53.4 & 8.2 & 1158.0 \\
\hline IE 7386 & Kenya & 3.88 & 71.6 & 110.5 & 4.4 & 86.7 & 51.8 & 8.1 & 1354.8 \\
\hline IE 4709 & Burundi & 3.86 & 64.2 & 113.9 & 8.1 & 124.7 & 47.4 & 8.3 & 464.0 \\
\hline Mean & & 4.30 & 76.8 & 102.1 & 5.3 & 101.2 & 52.5 & 7.7 & 1235.6 \\
\hline $\mathrm{SE} \pm$ & & 0.08 & 2.97 & 2.58 & 0.31 & 5.06 & 0.58 & 0.20 & 149.11 \\
\hline Least significant difference (LSD) & & 0.62 & & & & & & & \\
\hline Variance & & 0.10 & 132.51 & 99.68 & 1.45 & 384.38 & 5.05 & 0.58 & 333520.36 \\
\hline Range & & 1.03 & 36.4 & 32.8 & 4.1 & 63.9 & 8.7 & 2.3 & 1853.3 \\
\hline Minimum & & 3.86 & 59.9 & 87.9 & 4.0 & 62.7 & 47.4 & 6.7 & 464.0 \\
\hline \multirow[t]{2}{*}{ Maximum } & & 4.89 & 96.4 & 120.8 & 8.1 & 126.5 & 56.1 & 9.0 & 2317.3 \\
\hline & & & & & & & & & Controlcultivars \\
\hline IE 2043 (PR 202) & India & 2.71 & 71.4 & 98.3 & 5.1 & 76.4 & 54.0 & 7.7 & 2633.3 \\
\hline IE 3618 (RAU 8) & India & 3.08 & 65.4 & 95.0 & 5.0 & 78.1 & 52.1 & 7.7 & 2264.5 \\
\hline IE 4673 (VL 149) & India & 2.72 & 65.3 & 97.4 & 4.8 & 89.8 & 53.3 & 8.4 & 2002.6 \\
\hline VR 708 & India & 2.96 & 54.4 & 87.1 & 4.5 & 65.1 & 50.3 & 7.9 & 1553.3 \\
\hline Mean of control cultivars & & 2.87 & 64.1 & 94.5 & 4.9 & 77.4 & 52.5 & 7.9 & 2113.5 \\
\hline
\end{tabular}

Agronomic diversity assessment of the 15 best nutrients-dense accessions showed substantial variability as indicated by wide range and higher variances for selected agronomic traits within each set of the 15 best accessions for grain nutrient contents
(Tables 1-4). The best accessions rich in grain Ca content were significantly late to flower, taller and possessed longer and wider ear heads compared to those rich in Fe and $\mathrm{Zn}$ contents as indicated by two-sample paired ' $t$ ' test (Table 5). Similarly, the accessions rich in

Table 4

Performance of 15 best protein-rich finger millet core collection accessions for selected agronomic traits.

\begin{tabular}{|c|c|c|c|c|c|c|c|c|c|}
\hline Accession no. & Country & Protein (\%) & $\begin{array}{l}\text { Days to } 50 \% \\
\text { flowering }\end{array}$ & $\begin{array}{l}\text { Plant } \\
\text { height }(\mathrm{cm})\end{array}$ & Basal tillers & $\begin{array}{l}\text { Ear head } \\
\text { length } \\
(\mathrm{mm})\end{array}$ & $\begin{array}{l}\text { Ear head } \\
\text { width } \\
(\mathrm{mm})\end{array}$ & $\begin{array}{l}\text { Fingers per } \\
\text { ear head }\end{array}$ & $\begin{array}{l}\text { Grain yield } \\
\left(\mathrm{kg} \mathrm{ha}^{-1}\right)\end{array}$ \\
\hline IE 6537 & Nigeria & 11.09 & 86.6 & 90.8 & 4.1 & 98.8 & 53.7 & 7.6 & 762.6 \\
\hline IE 0009 & India & 10.44 & 74.0 & 108.2 & 4.1 & 67.6 & 54.0 & 7.7 & 1198.9 \\
\hline IE 4709 & Burundi & 9.95 & 64.2 & 113.9 & 8.1 & 124.7 & 47.4 & 8.3 & 464.0 \\
\hline IE 4708 & Burundi & 9.51 & 62.4 & 109.8 & 6.5 & 104.2 & 49.0 & 8.8 & 553.5 \\
\hline IE 6541 & Nigeria & 9.46 & 91.7 & 88.6 & 3.9 & 106.8 & 52.9 & 8.1 & 1183.3 \\
\hline IE 2921 & Malawi & 9.35 & 78.4 & 120.8 & 6.6 & 122.8 & 56.1 & 9.0 & 631.5 \\
\hline IE 6546 & Nigeria & 9.30 & 88.4 & 99.2 & 4.8 & 126.5 & 54.9 & 8.4 & 1464.6 \\
\hline IE 4476 & Zimbabwe & 9.01 & 72.1 & 91.7 & 4.6 & 104.5 & 52.5 & 8.3 & 543.2 \\
\hline IE 4443 & Cameroon & 8.81 & 70.0 & 87.9 & 4.6 & 62.7 & 52.4 & 7.3 & 2317.3 \\
\hline IE 588 & India & 8.80 & 51.4 & 81.5 & 5.1 & 53.9 & 51.3 & 7.4 & 1058.7 \\
\hline IE 6013 & Nepal & 8.80 & 61.5 & 92.9 & 4.3 & 66.7 & 54.3 & 8.1 & 803.0 \\
\hline IE 2093 & India & 8.79 & 56.1 & 80.2 & 4.8 & 57.6 & 50.5 & 6.2 & 1175.1 \\
\hline IE 4817 & India & 8.76 & 64.7 & 89.1 & 4.8 & 64.4 & 50.3 & 7.8 & 1173.8 \\
\hline IE 3120 & India & 8.72 & 62.9 & 91.6 & 3.9 & 71.5 & 53.9 & 7.8 & 1112.0 \\
\hline IE 3101 & India & 8.66 & 65.7 & 86.8 & 5.6 & 75.6 & 53.3 & 7.4 & 1628.6 \\
\hline Mean & & 9.30 & 70.0 & 95.5 & 5.0 & 87.2 & 52.4 & 7.9 & 1071.3 \\
\hline $\mathrm{SE} \pm$ & & 0.18 & 3.07 & 3.14 & 0.30 & 6.77 & 0.61 & 0.18 & 126.23 \\
\hline Least significant difference (LSD) & & 0.98 & & & & & & & \\
\hline Variance & & 0.51 & 141.09 & 147.93 & 1.39 & 687.20 & 5.51 & 0.47 & 239006.62 \\
\hline Range & & 2.43 & 40.3 & 40.6 & 4.2 & 72.7 & 8.7 & 2.8 & 1853.3 \\
\hline Minimum & & 8.66 & 51.4 & 80.2 & 3.9 & 53.9 & 47.4 & 6.2 & 464.0 \\
\hline Maximum & & 11.09 & 91.7 & 120.8 & 8.1 & 126.5 & 56.1 & 9.0 & 2317.3 \\
\hline \multicolumn{10}{|l|}{ Control cultivars } \\
\hline IE 2043 (PR 202) & India & 6.87 & 71.4 & 98.3 & 5.1 & 76.4 & 54.0 & 7.7 & 2633.3 \\
\hline IE 3618 (RAU 8) & India & 7.27 & 65.4 & 95.0 & 5.0 & 78.1 & 52.1 & 7.7 & 2264.5 \\
\hline IE 4673 (VL 149) & India & 7.14 & 65.3 & 97.4 & 4.8 & 89.8 & 53.3 & 8.4 & 2002.6 \\
\hline VR 708 & India & 7.91 & 54.4 & 87.1 & 4.5 & 65.1 & 50.3 & 7.9 & 1553.3 \\
\hline Mean of control cultivars & & 7.30 & 64.1 & 94.5 & 4.9 & 77.4 & 52.5 & 7.9 & 2113.5 \\
\hline
\end{tabular}


Table 5

Estimates of mean and variance among best 15 accessions for grain nutrients contents in a core collection of finger millet germplasm for selected quantitative traits.

\begin{tabular}{|c|c|c|c|c|c|c|c|c|c|c|c|c|c|}
\hline \multirow[t]{2}{*}{ Traits } & \multirow{2}{*}{$\begin{array}{l}\text { Comparison } \\
\text { Grain nutrient }\end{array}$} & \multicolumn{2}{|l|}{ Fe vs Zn } & \multicolumn{2}{|l|}{ Fe vs Ca } & \multicolumn{2}{|c|}{ Fe vs protein } & \multicolumn{2}{|l|}{ Zn vs Ca } & \multicolumn{2}{|c|}{ Zn vs protein } & \multicolumn{2}{|c|}{ Ca vs protein } \\
\hline & & $\mathrm{Fe}$ & $\mathrm{Zn}$ & $\mathrm{Fe}$ & $\mathrm{Ca}$ & $\mathrm{Fe}$ & Protein & $\mathrm{Zn}$ & $\mathrm{Ca}$ & $\mathrm{Zn}$ & Protein & $\mathrm{Ca}$ & Protein \\
\hline \multirow[t]{2}{*}{ Days to $50 \%$ flowering } & Mean & $65.04^{\mathrm{a}}$ & $69.60^{\mathrm{a}}$ & $62.85^{\mathrm{a}}$ & $80.51^{\mathrm{b}}$ & $65.13^{\mathrm{a}}$ & $74.44^{\mathrm{b}}$ & $68.00^{\mathrm{a}}$ & $76.32^{\mathrm{b}}$ & $69.01^{\mathrm{a}}$ & $69.51^{\mathrm{a}}$ & $78.66^{\mathrm{a}}$ & $66.00^{\mathrm{a}}$ \\
\hline & Variance & 69.76 & 55.22 & 74.40 & 142.40 & 64.60 & 159.20 & 31.99 & $140.97^{*}$ & 31.57 & $141.45^{*}$ & 165.40 & 152.60 \\
\hline \multirow[t]{2}{*}{ Plant height $(\mathrm{cm})$} & Mean & $96.01^{\mathrm{a}}$ & $100.10^{\mathrm{a}}$ & $91.59^{\mathrm{a}}$ & $100.78^{\mathrm{b}}$ & $94.28^{\mathrm{a}}$ & $93.40^{\mathrm{a}}$ & 99.40 & 101.70 & 101.81 & 96.10 & $102.23^{a}$ & $89.85^{b}$ \\
\hline & Variance & 162.95 & $30.40^{* *}$ & 97.37 & 57.94 & 83.59 & 49.65 & 25.88 & $109.61^{*}$ & ** $\quad 17.83$ & $181.53^{*}$ & 57.47 & 74.94 \\
\hline \multirow[t]{2}{*}{ Basal tillers (no.) } & Mean & $4.99^{\mathrm{a}}$ & $4.43^{\mathrm{a}}$ & 4.45 & 4.84 & $4.34^{\mathrm{a}}$ & $4.43^{\mathrm{a}}$ & $4.40^{\mathrm{a}}$ & $5.34^{\mathrm{b}}$ & 4.42 & 5.18 & $4.94^{\mathrm{a}}$ & $4.55^{\mathrm{a}}$ \\
\hline & Variance & 1.39 & $0.19^{*}$ & 0.24 & 0.73 & 0.22 & 0.35 & 0.21 & $1.60^{*}$ & 0.19 & $1.61^{*}$ & 0.84 & 0.38 \\
\hline \multirow[t]{2}{*}{ Ear head length (mm) } & Mean & $84.13^{\mathrm{a}}$ & $79.36^{\mathrm{a}}$ & $74.31^{\mathrm{a}}$ & $99.96^{\mathrm{b}}$ & $78.96^{\mathrm{a}}$ & $84.75^{\mathrm{a}}$ & $75.17^{\mathrm{a}}$ & $100.40^{\mathrm{b}}$ & 77.33 & 87.15 & $96.78^{\mathrm{a}}$ & $70.51^{\mathrm{b}}$ \\
\hline & Variance & 623.35 & 436.12 & 317.50 & 315.70 & 353.20 & 531.40 & 299.70 & 376.80 & 322.30 & 664.40 & 293.20 & 263.90 \\
\hline \multirow[t]{2}{*}{ Ear head width (mm) } & Mean & $51.52^{\mathrm{a}}$ & $52.29^{a}$ & $51.54^{\mathrm{a}}$ & $53.08^{\mathrm{a}}$ & $51.71^{\mathrm{a}}$ & $53.42^{\mathrm{a}}$ & $52.13^{\mathrm{a}}$ & $52.41^{\mathrm{b}}$ & 52.11 & 52.28 & $52.77^{\mathrm{a}}$ & $52.56^{\mathrm{a}}$ \\
\hline & Variance & 3.74 & 2.19 & 0.70 & 1.77 & 0.70 & 1.93 & 1.92 & 5.36 & 1.55 & $5.84 *$ & 1.62 & 2.64 \\
\hline \multirow[t]{2}{*}{ Fingers per ear head } & Mean & $7.54^{\mathrm{a}}$ & $7.50^{\mathrm{a}}$ & $7.15^{\mathrm{a}}$ & $7.40^{\mathrm{a}}$ & $7.24^{\mathrm{a}}$ & $7.90^{\mathrm{a}}$ & $7.38^{\mathrm{a}}$ & $7.65^{\mathrm{a}}$ & 7.37 & 7.86 & $7.30^{\mathrm{a}}$ & $7.60^{\mathrm{a}}$ \\
\hline & Variance & 0.91 & $0.28^{*}$ & 0.42 & 0.71 & 0.76 & $0.10^{*}$ & 0.21 & 0.62 & 0.24 & 0.56 & 0.37 & 0.39 \\
\hline \multirow[t]{2}{*}{ Grain yield $\left(\mathrm{kg} \mathrm{ha}^{-1}\right)$} & Mean & $1086.09^{a}$ & a $1550.94^{b}$ & b $1178.00^{a}$ & a $\quad 1402.00^{\mathrm{a}}$ & ${ }^{\mathrm{a}} 1194.00^{\mathrm{a}}$ & $1166.00^{a}$ & a $\quad 1573.00^{\mathrm{a}}$ & $a^{\mathrm{a}} \quad 1209.00^{\mathrm{a}}$ & $1626.00^{a}$ & a $\quad 1027.00^{b}$ & \multirow{2}{*}{\multicolumn{2}{|c|}{$\begin{array}{ccc}0^{\mathrm{b}} & 1475.00^{\mathrm{a}} & 1167.00^{\mathrm{a}} \\
123021 & 51666\end{array}$}} \\
\hline & Variance & 234451 & 98049 & 56635 & 146236 & 705058 & 86001 & 110053 & 382772 & 87496 & 286844 & & \\
\hline
\end{tabular}

Means with different letter superscripts indicate significant differences; * and **Significant differences in variances @P $\leq 0.05$ and @P $\leq 0.01$, respectively.

Ca content were significantly taller than those rich in protein content. The accessions rich in $\mathrm{Zn}$ content had significantly higher grain yield potential than those rich in Fe and protein contents; those rich in Ca content had numerically superior grain yield potential than those for Fe and protein contents (Table 5). The best accessions for grain nutrients did not differ significantly for other traits. The variances in days to $50 \%$ flowering, plant height and basal tillers differed significantly between $\mathrm{Zn}$ and $\mathrm{Ca}$ and between $\mathrm{Zn}$ and protein rich accessions (Table 5). Similarly, the variances in plant height, basal tillers and fingers per ear head differed significantly between low $\mathrm{Zn}$ and $\mathrm{Zn}$ rich accessions. The latter results are conclusively displayed in a principal component biplot (Fig. 1) for 24 accessions, which surpassed the mean of the control cultivars for two or more nutrients contents (Table 6).

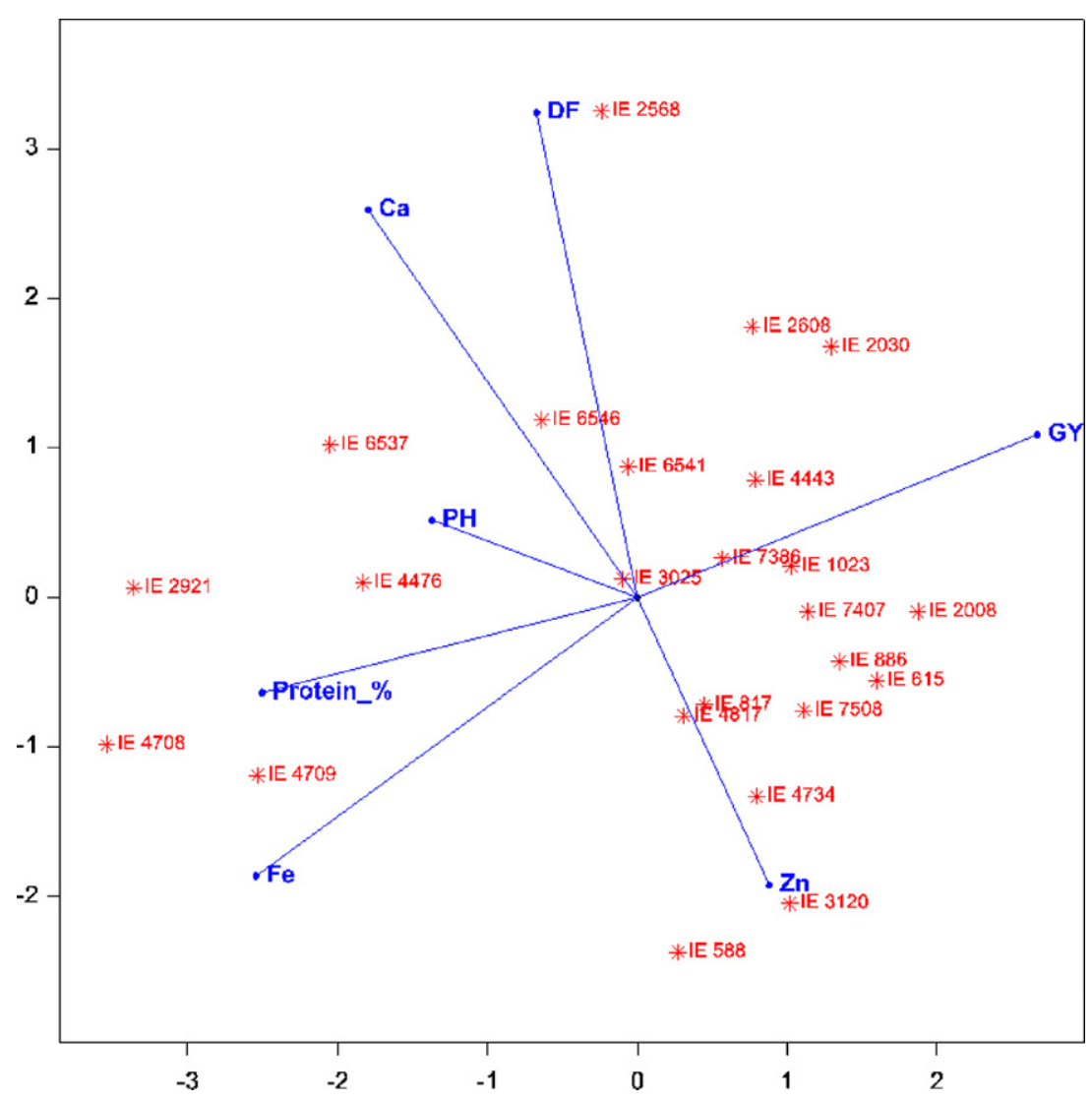

Fig. 1. Principal component biplot of 24 finger millet accessions (red asterisks) in regard to nutritional traits iron (Fe), calcium (Ca), Zink (Zn) and protein \% as well as grain yield (GY), days to $50 \%$ flowering (DF) and plant height (PH). (For interpretation of the references to colour in this figure legend, the reader is referred to the web version of the article.) 
Table 6

Performance of 24 accessions selected based on their superiority over mean of control cultivars for two or more grain nutrients in a core collection of finger millet.

\begin{tabular}{|c|c|c|c|c|c|c|c|c|c|c|c|c|}
\hline Accession No. & Country & $\begin{array}{l}\text { Days to } 50 \% \\
\text { flowering }\end{array}$ & $\begin{array}{l}\text { Plant } \\
\text { height } \\
(\mathrm{cm})\end{array}$ & $\begin{array}{l}\text { Basal } \\
\text { tillers }\end{array}$ & $\begin{array}{l}\text { Ear head } \\
\text { length } \\
(\mathrm{mm})\end{array}$ & $\begin{array}{l}\text { Ear head } \\
\text { width } \\
(\mathrm{mm})\end{array}$ & $\begin{array}{l}\text { Fingers ear } \\
\text { head }^{-1}\end{array}$ & $\begin{array}{l}\text { Grain yield } \\
\left(\mathrm{kg} \mathrm{ha}^{-1}\right)\end{array}$ & $\begin{array}{l}\mathrm{Fe} \\
\left(\mathrm{mg} \mathrm{kg}^{-1}\right)\end{array}$ & $\begin{array}{l}\mathrm{Zn} \\
\left(\mathrm{mg} \mathrm{kg}^{-1}\right)\end{array}$ & $\mathrm{Ca}\left(\mathrm{g} \mathrm{kg}^{-1}\right)$ & Protein (\%) \\
\hline IE 588 & India & 51.4 & 81.5 & 5.1 & 53.9 & 51.3 & 7.4 & 1058.7 & 45.91 & 21.85 & 2.84 & 8.80 \\
\hline IE 615 & India & 65.0 & 102.2 & 4.5 & 76.0 & 51.1 & 6.9 & 1977.5 & 35.38 & 23.17 & 3.15 & 7.67 \\
\hline IE 817 & India & 66.9 & 100.1 & 5.3 & 78.9 & 50.2 & 7.2 & 1239.7 & 37.66 & 21.88 & 3.00 & 8.17 \\
\hline IE 886 & Pakistan & 67.2 & 99.2 & 5.0 & 72.4 & 52.1 & 7.1 & 1729.4 & 33.31 & 22.53 & 3.06 & 7.95 \\
\hline IE 1023 & Unknown & 73.5 & 97.5 & 3.7 & 69.5 & 53.4 & 7.5 & 1468.9 & 33.04 & 22.66 & 3.55 & 7.55 \\
\hline IE 2008 & India & 67.8 & 98.6 & 4.8 & 69.6 & 51.5 & 6.6 & 2095.7 & 33.07 & 22.78 & 3.26 & 7.53 \\
\hline IE 2030 & India & 64.8 & 94.0 & 5.0 & 70.6 & 53.4 & 7.4 & 1958.2 & 28.15 & 19.98 & 4.69 & 7.10 \\
\hline IE 2568 & Kenya & 96.4 & 101.9 & 4.1 & 103.8 & 52.4 & 6.8 & 1031.3 & 25.81 & 19.25 & 4.51 & 7.41 \\
\hline IE 2608 & Malawi & 79.4 & 100.9 & 5.4 & 115.5 & 54.5 & 6.7 & 1990.9 & 32.25 & 20.73 & 4.24 & 7.72 \\
\hline IE 2921 & Malawi & 78.4 & 120.8 & 6.6 & 122.8 & 56.1 & 9.0 & 631.5 & 59.09 & 20.81 & 4.19 & 9.35 \\
\hline IE 3025 & Ethiopia & 81.8 & 109.7 & 4.1 & 131.0 & 53.2 & 7.8 & 1061.1 & 33.25 & 23.63 & 3.38 & 8.52 \\
\hline IE 3120 & India & 62.9 & 91.6 & 3.9 & 71.5 & 53.9 & 7.8 & 1112.0 & 34.29 & 25.33 & 2.80 & 8.72 \\
\hline IE 4443 & Cameroon & 70.0 & 87.9 & 4.6 & 62.7 & 52.4 & 7.3 & 2317.3 & 37.90 & 20.98 & 4.15 & 8.81 \\
\hline IE 4476 & Zimbabwe & 72.1 & 91.7 & 4.6 & 104.5 & 52.5 & 8.3 & 543.2 & 44.94 & 22.44 & 4.89 & 9.01 \\
\hline IE 4708 & Burundi & 62.4 & 109.8 & 6.5 & 104.2 & 49.0 & 8.8 & 553.5 & 65.23 & 20.76 & 4.51 & 9.51 \\
\hline IE 4709 & Burundi & 64.2 & 113.9 & 8.1 & 124.7 & 47.4 & 8.3 & 464.0 & 48.56 & 22.44 & 3.86 & 9.95 \\
\hline IE 4734 & India & 53.5 & 82.2 & 4.4 & 56.2 & 53.1 & 6.1 & 1170.1 & 39.63 & 20.35 & 3.03 & 8.01 \\
\hline IE 4817 & India & 64.7 & 89.1 & 4.8 & 64.4 & 50.3 & 7.8 & 1173.8 & 36.29 & 22.46 & 3.47 & 8.76 \\
\hline IE 6537 & Nigeria & 86.6 & 90.8 & 4.1 & 98.8 & 53.7 & 7.6 & 762.6 & 34.41 & 21.49 & 4.39 & 11.09 \\
\hline IE 6541 & Nigeria & 91.7 & 88.6 & 3.9 & 106.8 & 52.9 & 8.1 & 1183.3 & 32.19 & 20.93 & 3.05 & 9.46 \\
\hline IE 6546 & Nigeria & 88.4 & 99.2 & 4.8 & 126.5 & 54.9 & 8.4 & 1464.6 & 36.23 & 23.94 & 4.61 & 9.30 \\
\hline IE 7386 & Kenya & 71.6 & 110.5 & 4.4 & 86.7 & 51.8 & 8.1 & 1354.8 & 32.27 & 23.48 & 3.88 & 7.51 \\
\hline IE 7407 & Kenya & 75.5 & 103.4 & 4.3 & 64.8 & 54.1 & 7.3 & 1468.7 & 34.22 & 23.31 & 3.18 & 7.38 \\
\hline IE 7508 & Ethiopia & 66.9 & 101.9 & 4.0 & 79.6 & 51.7 & 8.1 & 1338.0 & 33.92 & 24.16 & 3.33 & 7.52 \\
\hline Mean & & 71.8 & 98.6 & 4.8 & 88.1 & 52.4 & 7.6 & 1297.9 & 37.79 & 22.14 & 3.71 & 8.45 \\
\hline $\mathrm{SEM} \pm$ & & 2.30 & 2.01 & 0.20 & 4.97 & 0.39 & 0.15 & 105.53 & 1.86 & 0.30 & 0.14 & 0.20 \\
\hline Variance & & 127.12 & 96.89 & 1.00 & 591.65 & 3.70 & 0.52 & 267299.04 & 83.46 & 2.19 & 0.44 & 0.99 \\
\hline Range & & 44.9 & 39.3 & 4.4 & 77.1 & 8.7 & 2.9 & 1853.3 & 39.42 & 6.08 & 2.09 & 3.99 \\
\hline Minimum & & 51.4 & 81.5 & 3.7 & 53.9 & 47.4 & 6.1 & 464.0 & 25.81 & 19.25 & 2.80 & 7.10 \\
\hline Maximum & & 96.4 & 120.8 & 8.1 & 131.0 & 56.1 & 9.0 & 2317.3 & 65.23 & 25.33 & 4.89 & 11.09 \\
\hline \multicolumn{13}{|c|}{ Controlcultivars } \\
\hline IE 2043 (PR 202) & India & 71.4 & 98.3 & 5.1 & 76.4 & 54.0 & 7.7 & 2633.3 & 26.51 & 18.89 & 2.71 & 6.87 \\
\hline IE 3618 (RAU 8) & India & 65.4 & 95.0 & 5.0 & 78.1 & 52.1 & 7.7 & 2264.5 & 31.49 & 19.71 & 3.08 & 7.27 \\
\hline IE 4673 (VL 149) & India & 65.3 & 97.4 & 4.8 & 89.8 & 53.3 & 8.4 & 2002.6 & 35.99 & 21.39 & 2.72 & 7.14 \\
\hline VR 708 & India & 54.4 & 87.1 & 4.5 & 65.1 & 50.3 & 7.9 & 1553.3 & 30.19 & 19.57 & 2.96 & 7.91 \\
\hline Mean of control cultivars & & 71.4 & 98.3 & 5.1 & 76.4 & 54.0 & 7.7 & 2633.3 & 31.045 & 19.89 & 2.87 & 7.30 \\
\hline
\end{tabular}

Correlation coefficients of grain yield with $\mathrm{Fe}(0.03), \mathrm{Zn}(0.05), \mathrm{Ca}$ (0.001) and protein (0.09) contents were weak and non-significant. Though correlation coefficients of Fe with $\mathrm{Zn}(0.28, P<0.05)$ and protein $(0.25, P<0.05)$ and that of $\mathrm{Zn}$ with protein $(0.16, P<0.05)$ contents were statistically significant, the magnitudes were lower.

Grouping of 24 accessions which surpassed mean of the control cultivars for two or more nutrients contents (Table 6) resulted in four clusters. The number of accessions varied from three (in cluster I) to 11 (in cluster IV) (Fig. 2). While the clusters I and III had accessions only from Africa, Cluster II and cluster IV consisted of accessions from Africa and India. Only mean days to 50\% flowering and mean number of fingers per ear head differed between the clusters. The accessions included in cluster I were significantly late to flower (90 days) than those included in other clusters (68.4 days in cluster II, 68.3 days in cluster III and 64.2 days in cluster IV). The accessions of cluster III produced significantly higher number of fingers per ear head (8.7) than those of other clusters ( 7.8 in cluster I, 7.2 in cluster II and 7.7 in cluster IV). The variances of quantitative traits did not differ among the clusters.

The relationships of mean grain nutrient contents with qualitative traits were investigated. The accessions with green plants $\left(2.9 \mathrm{~g} \mathrm{~kg}^{-1}\right)$ and prostrate growth habit $\left(3.7 \mathrm{~g} \mathrm{~kg}^{-1}\right)$ and having elongata type of ear heads $\left(3.1 \mathrm{~g} \mathrm{~kg}^{-1}\right)$ and reddish brown/ragi brown/dark brown grains (2.9, 3.0 and $2.8 \mathrm{~g} \mathrm{~kg}^{-1}$ ) had significantly higher Ca content than those with purple plants $\left(2.7 \mathrm{~g} \mathrm{~kg}^{-1}\right)$ and erect/decumbent growth habit (2.8 and $\left.3.0 \mathrm{~g} \mathrm{~kg}^{-1}\right)$ having plana/vulgaris/compacta types of ears $\left(2.9,2.8\right.$ and $2.8 \mathrm{~g} \mathrm{~kg}^{-1}$ ) and light brown/white grains $\left(2.9 \mathrm{~g} \mathrm{~kg}^{-1}\right)$. The accessions with prostrate type of growth habit $\left(36.0 \mathrm{mg} \mathrm{kg}^{-1}\right)$ also had signifi- cantly higher Fe content than those with either erect/decumbent type (29.16 and $29.4 \mathrm{mg} \mathrm{kg}^{-1}$ ). This was more pronounced in six wild accessions, which had higher mean grain Fe content (47.6 $\mathrm{mg} \mathrm{kg}^{-1}$ ) with prostrate type of growth habit. The accessions bearing dark brown/reddish brown/ragi brown grains (7.5, 7.4 and $7.6 \%)$ had significantly higher protein content than those bearing light brown/white grains (7.2 and 7.0\%).

The mean grain nutrient contents of accessions differing in geographical origins were comparable (data not given).

\section{Discussion}

\subsection{Variability for grain nutrient contents}

Analysis, detection and exploitation of existing genetic variability is the short-term strategy for identification of finger millet genotypes rich in grain nutrients to meet the immediate requirement of target micronutrient and protein deficient populations. Substantial variability for all the four grain nutrients observed in the finger millet core collection suggest ample scope for the selection of nutrient-rich accessions for use in the breeding programmes. The accessions such as IE 4708, IE 2921, IE 4709, IE 588, IE 5736, IE 4476 and IE 942 rich in Fe content ( 40.60 to $65.23 \mathrm{mg} \mathrm{kg}^{-1}$ ); IE 3120 , IE 7508, IE 6546, IE 3025 and IE 7386 rich in Zn content (23.48 to $25.33 \mathrm{mg} \mathrm{kg}^{-1}$ ); IE 4476, IE 2030, IE 6546 IE 4708, IE 2568, IE 2957 and IE 6537 rich in Ca content (4.39 to $4.89 \mathrm{~g} \mathrm{~kg}^{-1}$ ); IE 6537, IE 0009, IE 4709, IE 4708, IE 6541, IE 2921, IE 6546 and IE 4476 rich in protein content (9.01-11.09\%), surpassed trial mean of respective grain nutrient contents +1 LSD units. A few of the accessions were 


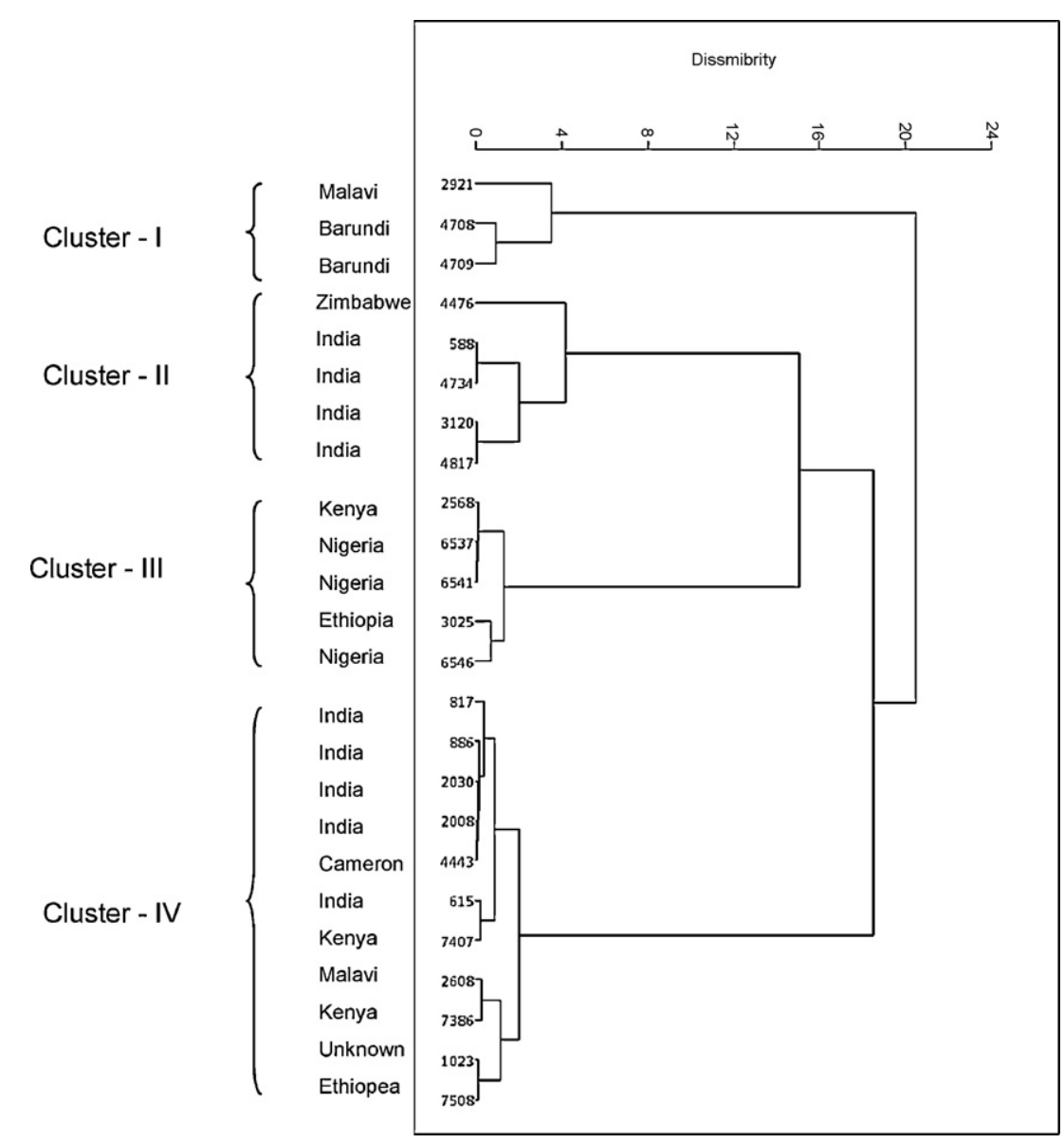

Fig. 2. Dendrogram showing clustering pattern of 24 finger millet accessions selected based on their superiority over mean of the control cultivars for at least two grain nutruents contents in a core collection of finger millet germplasm.

significantly higher in more than one grain nutrient contents. For example, the accessions, IE 4708, IE 4476 and IE 6546 are rich in $\mathrm{Fe}, \mathrm{Ca}$ and protein contents and IE 6537 is rich in Ca and protein contents. These accessions could be involved in hybridization with agronomically superior accessions/breeding lines to combine grain nutrients and farmer and consumer preferred traits.

Past attempts by Kempanna and Kavallappa (1968) and Shashi et al. (2007) to detect and estimate genetic variability for grain nutrients in finger millet were based on a relatively fewer numbers of accessions (20 and 30, respectively), which represent limited variability of the world's finger millet accessions. All the reported genetic variability for grain micronutrients and protein contents in finger millet are among the cultivars bred for grain and fodder yields. The grain nutrient contents in such bred cultivars do not represent the true genetic variability as there is possible decline in grain nutrient contents as a result of the correlated response to selection for grain and fodder yields as reported in wheat (Garvin et al., 2006). Welch and Graham (2004) and Graham et al. (2001) have also reported that as progress is made for crop yield, there is concomitant reduction in mineral contents in edible parts of grain crops.

The variability for grain nutrients reported in the present study is from a core collection constituted from an entire collection of world's finger millet germplasm (Upadhyaya et al., 2006). Large variability among core collection accessions offers ample scope for the selection of micronutrient and protein-dense finger millet accessions. Such accessions could be released for commercial cultivation if found acceptable for agronomic traits as well after extensive testing to meet the immediate requirement of target populations. Analysis and exploitation of hybridization-derived variability are the next step to combine high grain nutrient density and desirable agronomic traits.

Considering that finger millet is grown predominantly as a rainfed crop on different soil types and associated differences in the native soil fertility with/without farmers' managed fertility in India, it is necessary to assess the stability of macro and micronutrients and protein-dense finger millet cultivars across different soil types and soil fertility levels typical of the areas to which these cultivars are targeted. Knowledge on genotype $\times$ environment interaction aids in designing suitable breeding and selection strategies to enhance nutrients in edible portions of grain (Pfeiffer and McClafferty, 2007). Introduction of micronutrient and proteindense finger millet cultivars would help reduce malnutrition due to micronutrients and protein deficiency of resource-poor people who consume finger millet-based diet in large quantities on a daily basis.

Weaker and non-significant correlation coefficients of grain yield with $\mathrm{Fe}, \mathrm{Zn}, \mathrm{Ca}$ and protein contents, and statistically significant but lower magnitude of correlation coefficients of Fe with $\mathrm{Zn}$ and protein contents and that of $\mathrm{Zn}$ with protein contents suggest better prospects of combining higher grain nutrients with higher grain yield potential.

Although it was known that beta-carotene content is low in finger millet (Kandlakunta et al., 2008), seed samples of the core collection were analyzed to detect genotypes with exceptional beta-carotene content. However in general, results showed very low beta-carotene contents and no variation for the trait in the core collection. 


\subsection{Heritability}

The heritability estimates indicate relative importance of genetic variation to the total variation in a population and hence they depend on the absolute size of genetic and environmental variations; the former varies with the type of population [individuals, half-sibs, full-sibs, selfed $\left(\mathrm{S}_{1}, \mathrm{~S}_{2}\right)$ progenies] while the latter is dependent on the experimental conditions and number of replications in which the traits are measured (Holland et al., 2003; Hallauer, 2007). Heritability estimates for traits measured in different populations and environments will seldom be quantitatively comparable although they could be qualitatively similar. In the present study, broad-sense heritability estimates were relatively higher for $\mathrm{Ca}$ and protein contents (as reflected by relatively narrow difference between PCV and GCV), and low for $\mathrm{Zn}$ and Fe contents (as reflected by relatively wider difference between PCV and GCV). Differential influence of the crop environment (which include weather variables and native and managed soil-fertility levels) relative to the genetic potential of the accessions to accumulate nutrients could have resulted in differences in heritability estimates.

Relative magnitude of additive (fixable) and non-additive (non-fixable) genetic variation is confounded in the estimates of broad-sense heritability (Roy, 2000; Chahal and Gosal, 2002). Since finger millet is predominantly self pollinated crop, the germplasm represents a mixture of pure-lines (Falconer and Mackay, 1996). The genetic component of variability among finger millet germplasm accessions is therefore attributable to additive and additive-based epistatic interaction of genes controlling different grain nutrients contents. Hence, the broad-sense heritability estimates for different traits in the present study are indicative of narrow-sense heritability estimates. Genotype $\times$ environment interaction could also be confounded with heritability estimates as they are based on a single season data. Nevertheless, as the genetic variations for grain nutrients among the finger millet germplasm accessions are solely attributable to genes acting additively, causing greater resemblance between selected parents and their progeny, higher genetic advance could be realized even with simple purelines selection.

\subsection{Agronomic diversity of the 15 best nutrients-dense accessions}

The BLUPs of quantitative traits based on the data pooled from five locations were used for assessing agronomic diversity of the 15 best nutrients-dense accessions. The magnitudes of range and variance of selected agronomic traits within each set of the 15 best accessions for grain nutrients contents were substantial. The best accessions rich in Ca content were significantly late to flower, taller and possess longer and wide ear heads compared to those rich in $\mathrm{Fe}$ and $\mathrm{Zn}$ contents. Similarly, the accessions rich in Ca content were significantly taller than those rich in protein content. The accessions rich in $\mathrm{Zn}$ have significantly higher grain yield potential than those rich in Fe and protein content; those rich in Ca content have numerically superior grain yield potential than those rich in Fe and protein contents. The best accessions for grain nutrients did not differ significantly for other traits.

The variances for days to $50 \%$ flowering, plant height and basal tillers differed significantly between $\mathrm{Zn}$ and $\mathrm{Ca}$ and between $\mathrm{Zn}$ and protein rich accessions. Similarly, the variances for plant height, basal tillers and fingers per ear head differed significantly between $\mathrm{Fe}$ and $\mathrm{Zn}$ rich accessions. These results suggest that the genotypes which accumulate higher grain $\mathrm{Zn}$ and Ca have tendency for higher grain yield potential than those which accumulate higher Fe and protein. Alternatively, the genotypes accumulate Fe and protein contents at the cost of grain yield potential. The seeds of cultivars rich in $\mathrm{Zn}$ are known to confer agronomic advantages such as higher seedling vigour, higher levels of resistance to diseases besides empowering the crop with higher water-use efficiency (Cakmak et al., 1999; Graham and Welch, 1996), all of which are decisive and critical benefits in the semi-arid tropics, where finger millet is grown in large area.

The 24 accessions rich at least in two grain nutrients could be grouped into four clusters based on the diversity of agronomic traits. The accessions sharing common geographical origin were grouped into a single cluster (Fig. 2). Days to 50\% flowering, plant height and grain yield per plant were the key traits which differentiated these 24 accessions best in at least two nutrients (Fig. 1). Mean days to $50 \%$ flowering and number of fingers per ear head differed significantly among the clusters. As finger millet has evolved in regions characterized by large biodiversity (Hilu and de Wet, 1976a) and spread to the regions with diverse climatic conditions due to sea trade and anthropogenic activities (Dida et al., 2008), it is possible that vast differences in natural selection pressure might have resulted in significant differences in days to 50\% flowering and fingers per ear head (a contributing trait to grain yield per plant). Days to $50 \%$ flowering play an important role in the adaptation of genotypes for matching crop cycle with differential rainfall and distribution pattern prevailing in the geographical regions, from where the accessions originated. The number of fingers per ear head is associated with reproductive fitness on which the natural selection operates.

\subsection{Relationship of grain nutrient contents with quantitative traits}

Results illustrated in the biplot (Fig. 1) imply a trade-off between grain yield and protein content, which is in agreement with findings of Simmonds (1996) who reported a negative correlation between grain yield and protein for all major cereal crops. Furthermore, the biplot shows that finger millet genotypes with high Fe content were rarely found in combination with high grain yield and Ca rich genotypes were not found in combination with high $\mathrm{Zn}$ contents. However, the high degree of variation among the accessions best in each of the nutrients together with statistically significant but weaker correlation among the nutrients and with agronomic traits suggested that it is possible to develop nutrient-rich finger millet cultivars with a range of maturity duration to match rainfall duration and distribution pattern and other traits such as basal tillers and plant height, which contribute to grain and stover yields.

\subsection{Relationship of grain mutrient contents with qualitative traits}

Grain nutrient assessment in large segregating populations in routine breeding programmes is not feasible owing to laborious and expensive protocol. Identification of simply inherited easily observable morphological marker traits such as plant pigmentation, growth habit, ear head compactness and shape and grain colour, which are closely associated with grain nutrient contents would serve as surrogates for indirect selection of grain nutrientdense genotypes. Association of simply inherited morphological traits such as grain colour in common bean with quantitative traits such as grain size has been established by Sax (1923) and Rasmusson (1933) and analytically elaborated by Thoday (1961) and Law (1967).

In the present study, accessions grouped by different qualitative traits (growth habit, plant pigmentation, ear head compactness and shape and grain colour) significantly differed for the mean grain $\mathrm{Fe}, \mathrm{Ca}$ and protein contents. However, marginal differences in grain nutrient contents limit the use of these qualitative traits as surrogates for selecting finger millet lines rich in grain nutrient contents. 
4.6. Relationship of grain nutrient contents with geographical origin

The information on the relationship of grain nutrient contents with geographical origin provides clues for designing strategies for planning and collecting grain nutrient-specific germplasm. A comparison of mean grain nutrients contents of the accessions classified by geographic origin indicated poor evidence for the relationship of grain nutrient contents with geographical origin. The accessions with both high and low grain nutrient contents are equally likely to be present in Africa and Asia, the primary and secondary centers of origin of finger millet (Hilu and de Wet, 1976a; Dida et al., 2008).

\subsection{Grain nutrient-specific accessions}

The efficiency and pace of breeding finger millet for enhanced grain nutrient contents hinges on the precise information on magnitude of fixable (additive and additive-based epistasis) component of genetic variability, $g \times \mathrm{e}$ (both spatial and temporal) interaction and inheritance pattern of grain nutrient contents. The identification of accessions contrasting for grain nutrient contents is a prerequisite for inheritance and $\mathrm{g} \times \mathrm{e}$ interaction studies and developing appropriate populations for DNA marker-assisted tagging and/or mapping genomic regions causing variation in grain nutrient contents.

The accessions such as IE 3270, IE 3392 and IE 6300 (with $<23 \mathrm{mg} \mathrm{kg}^{-1}$ Fe content); IE 2921, IE 4708 and IE 4709 (with $>47 \mathrm{mg} \mathrm{kg}^{-1}$ Fe content); IE 3693, IE 4476 and IE 7128 (with $<17 \mathrm{mg} \mathrm{kg}^{-1} \mathrm{Zn}$ content) and IE 3120, IE 6546 and IE 7508 (with $>23 \mathrm{mg} \mathrm{kg}^{-1} \mathrm{Zn}$ content); IE 5812, IE 6300 and IE 6852 (with $<2 \mathrm{~g} \mathrm{~kg}^{-1}$ Ca content) and IE 2030, IE 4476 and IE 6546 (with $>4 \mathrm{~g} \mathrm{~kg}^{-1}$ Ca content); IE 3363, IE 5475 and IE 7390 (with $<6.5 \%$ protein content); IE 0009, IE 4709 and IE 6537 (with higher $>9.5 \%$ protein content) (Tables 1-4) are useful for such studies. The accessions, IE 588, IE 2921, IE 4443, IE 4476, IE 4817, IE 4708, IE 4709, and IE 6546 high in all the grain nutrient contents (Tables 1-4) and those (IE 3270, IE 3392, IE 4257, IE 6300, IE 6652 and IE 6996) low in all the grain nutrient contents are useful for developing planned crosses for unravelling the inheritance pattern and developing multiple grain nutrient content-based mapping populations for chromosomal localization and deciphering mode of action of genes controlling all the four grain nutrients simultaneously. These accessions contrasting for all the nutrients are also useful in making crosses with well-adapted and/or stable high yielding cultivars to generate variability for selecting nutrient-rich genotypes with adaptive and productive traits.

\section{Acknowledgements}

The authors gratefully acknowledge the financial support of the BMZ/GTZ project on "Sustainable conservation and utilization of genetic resources of two underutilized crops-finger millet and foxtail millet- to enhance productivity, nutrition and income in Africa and Asia" funded by the Federal Ministry for Economic Cooperation and Development (BMZ), Germany to carry out this activity.

\section{References}

Cakmak, I., Kalayci, M., Ekiz, H., Braun, HJ., Kilinc, Y., Yilmaz, A., 1999. Zinc deficiency as a practical problem in plant and human nutrition in Turkey: a NATO-science for stability project. Field Crops Res. 60, 175-188.

Chahal, G.S., Gosal, S.S., 2002. Principles and Procedures of Plant Breeding: Biotechnological and Conventional Approaches. Narosa Publishing House, New Delhi, India, p. 604.

Chand, R., Kumar, P., Sinha, S., 2003. Impact of Agricultural Trade and Related Reforms on Domestic Food Security in India. Institute of Economic Growth, New Delhi, India, p. 116.
Chennaveeraiah, M.S., Hiremath, S.C., 1973. Genome relationships of Eleusine tristachya and E. floccifolia. J. Cytol. Genet. 8, 1-5

Chennaveeraiah, M.S., Hiremath, S.C., 1974. Genome analysis of Eleusine coracana (L.) Gaertn. Euphytica 23, 489-495.

Chethan, S., Malleshi, N.G., 2007. Finger millet poly phenols: characterization and their nutraceutical potential. Am. J. Food Technol. 2, 618-629.

Dendy, D.A.V., 1993. Opportunities for non-traditional uses of the minor millets. In: Riley, K.W., Gupta, S.C., Seetharam, A., Mushonga, J.N. (Eds.), Advances in Small millets. Oxford \& IBH Publishing Co., New Delhi, India, pp. 259-270.

Dida, M.M., Wanyera, N., Dunn, M.L.H., Bennetzen, J.L., Devos, K.M., 2008. Population structure and diversity in finger millet (Eleusine coracana) germplasm. Trop. Plant Biol. 1, 131-141.

Falconer, D.S., Mackay, T.F.C., 1996. Introduction to Quantitative Genetics, fourth ed. Pearson Education Limited, Edinburgh Gate, Harlow, England, p. 464.

Federer, W.T., 1961. Augmented designs with one-way elimination of heterogeneity. Biometrics 17, 447-473.

Food and Agriculture Organization, 2004, http://www/FAO.ORG/FAOSTAT database.

Garvin, D.F., Welch, R.M., Finley, J.W., 2006. Historical shifts in the seed mineral micronutrient concentration of US hard red winter wheat germplasm. J. Sci. Food Agric. 86, 2213-2220.

Gibson, R.S., Hess, S.Y., Hotz, C., Brown, K.H., 2008. Indicators of zinc status at the population level: a review of the evidence. Br. J. Nutr. 99 (Suppl. 3), S14-S23.

Gopalan, C., Rama Sastri, B.V., Balasubramanian, S.C., 2002. Nutritive Value of Indian Foods. National Institute of Nutrition, Indian Council of Medical Research, Hyderabad, India.

Graham, R.D., Welch, R.M., 1996. Breeding for staple food crops with high micronutrient density. Agricultural Strategies for Micronutrients. Working Paper 3, Washington DC, USA. International Food Policy Research Institute, USA.

Graham, R.D., Welch, R.M., Bouis, H.E., 2001. Addressing micronutrient malnutrition through enhancing nutritional quality of staple foods: principles, perspectives and knowledge gaps. Adv. Agron. 70, 77-142.

Graham, R.D., Welch, R.M., Saunders, D.A., Ortiz Monasterio, I., Bouis, H.E., Bonierbale, M., de Haan, S., Burgos, G., Thiele, G., Liria, R., Meisner, C.A., Beebe, S.E., Potts, M.J., Kadian, M., Hobbs, P.R., Gupta, R.K., Twomlow, S., 2007. Nutritious subsistence food systems. Adv. Agron. 92, 1-74.

Hallauer, A.R., 2007. History, contribution, and future of quantitative genetics in plant breeding: lessons from maize. Crop Sci. 47 (S3), S5-S19.

Hardy, R.J., Thompson, S.G., 1996. A likelihood approach to meta-analysis with random effects. Stat. Med. 15, 619-629.

Hilu, K.W., de Wet, J.M.J., 1976a. Domestication of Eleusine coracana. Econ. Bot. 30, 199-208.

Hilu, K.W., de Wet, J.M.J., 1976b. Racial evolution in Eleusine coracana ssp. Coracana (Finger millet). Am. J. Bot. 63, 1311-1318.

Hiremath, S.C., Salimath, S.S., 1992. The 'A' genome donor of Eleusine coracana (L) Gaertn (Gramineae). Theor. Appl. Genet. 84, 747-754.

Holland, J.B., Nyquist, W.E., Cervantes-Martinez, C.T., 2003. Estimating and interpreting heritability for plant breeding: an update. Plant Breed. Rev. 22, 9-111.

IBPGR, 1985. Descriptors for Finger Millet. International Board for Plant Genetic Resources, Secretariat, Rome, p. 20.

Iyengar, K.G., Doraisami, L.S., Iyengar, R.S., 1945. Ragi (Eleusine coracana). Mysore J. Agric. Sci. 24, 33.

Kandlakunta, B., Rajendran, A., Thingnganing, L., 2008. Carotene content of some common (cereals, pulses, vegetables, spices and condiments) and unconventional sources of plant origin. Food Chem. 106, 85-89.

Kempanna, C., Kavallappa, B.N., 1968. Quantitative Assessment for nutritive quality of Eleusine Coracana (finger millet or ragi). Mysore J. Agric. Sci. 2, 324-329.

Keuls, M., 1952. The use of "studentized range" in connection with an analysis of variance. Euphytica 1, 112-122.

Krishnaswamy, K., 2009. The problem of double burden of disease and its consequences-a brief overview. In: Programme and abstracts, Symposium on Nutritional Security for India-Issues and Way Forward, Indian National Science Academy, New Delhi, 3-4 August, pp. 5-6.

Law, C.N., 1967. The location of genetic factors controlling a number of quantitative characters in wheat. Genetics 56, 445-461.

Levene, H., 1960. Robust tests for equality of variances. In: Olkin, I. (Ed.), Contributions to Probability and Statistics: Essays in Honor of Harold Hotelling. Stanford University Press, Stanford, USA, pp. 278-292.

Malleshi, N.G., Klopfenstein, C.F., 1998. Nutrient composition, amino acid and vitamin contents of malted sorghum, pearl millet, finger millet and their rootlets. Int. J. Food Sci. Nutr. 49, 415-422.

Mnyenyembe, P.H., Gupta, S.C., 1998. Variability for grain yield and related traits in finger millet germplasm accessions from Malawi. Afr. Crop Sci. J. 6, 317322 .

Newman, D., 1939. The distribution of range in samples from a normal population expressed in terms of an independent estimate of standard deviation. Biometrika 31, 20-30.

Obilana, A.B., Manyasa, E.O., Kibuka, J.G., Ajanga, S., 2002. Finger Millet Blast Samples Collection in Kenya: Passport Data, Analyses of Disease Incidence and Report of Activities. NARO, Uganda-ICRISAT-HRI, UK-KARI, Kakamega, ICRISAT-Nairobi, $12 \mathrm{pp}$.

Paterson, H.D., Williams, E.R., 1976. A new class of resolvable incomplete block designs. Biometrika 63, 83-92.

Payne, R.W., Senn, S., 2007. GenStat for Windows, 10th ed. Combines Estimates from Individual Trials (http://www.vsni.co.uk).

Pearson, K., 1901. On lines and planes of closest fit to a system of points in space. Philos Mag 2, 557-572. 
Pfeiffer, W.H., McClafferty, B., 2007. HarvestPlus: breeding crops for better nutrition. Crop Sci. 47, S88-S105.

Prasad, Rajendra, 2010.Zinc biofortification of food grains in relation to food security and alleviation of zinc malnutrition. Curr. Sci. 98, 1300-1304.

Rasmusson, J.M., 1933. A contribution to the theory of quantitative character inheritance. Hereditas 18, 245-261.

Roy, D., 2000. Plant Breeding: The Analysis and Exploitation of Variations. Narosa Publishing House, New Delhi, India.

Sahrawat, K.L., Ravi Kumar, G., Rao, J.K., 2002. Evaluation of triacid and dry ashing procedures for determining potassium, calcium, magnesium, iron, zinc, manganese and copper in plant materials. Commun. Soil Sci. Plant Anal. 33, 95-102.

Sax, K., 1923. The association of size differences with seed coat pattern and pigmentation in Phaseolus vulgaris. Genetics 8, 552-560.

Schonfeld, P., Werner, H.J., 1986. Beitragr zur teorie and anwendung linearer midelle. In: Krelle, W. (Ed.), Okonomische progress-entscheidungsund gleichgewichtsmodelle. VCH Verlagsgesellschaft, Weinheim, pp. 251-262.

Seetharam, A., Patel, D.P., Halaswamy, B.H., 2006. Small millets. In: Dhillon, B.S. Saxena, S., Agrawal, A., Tyagi, R.K. (Eds.), Plant Genetic Resources: Foodgrain Crops. Narosa Publishing Co. Ltd., New Delhi, India, pp. 204-222.

Senthil, N., Kumari, A., Nirmala, Joel, A., John, Selvi, B., Raveendran, T.S., Ramamoorthy, K., Ramanathan, A., 2005. Small Millets for Nutritional Security, first ed. Kalaiselvam Pathipagam, Coimbatore, Tamil Nadu, India.

Shashi, B.K., Sharan, S., Hittalmani, S., Shankar, A.G., Nagarathan, K.T., 2007. Micronurtient composition, Anti nutritional factors and Bioaccessibility of Iron in different finger millet (Eleusine coracana) genotypes. Karnataka J. Agric. Sci. 20, 583-585.

Simmonds, N.W., 1996. Yields of cereal grain and protein. Exp. Agric. 32, 351-356.

Singh, U., Jambunathan, R. 1980. Evaluation of rapid methods for the estimation of protein in chickpea (Cicer areietinum L.). J. Sci. Food Agric. 31, 247-254.
Snedecor, G.W., Cochran, W.G., 1994. Statistical Methods, eighth ed. Iowa State University Press, Ames, Iowa, USA.

Thoday, J.M., 1961. Location of polygenes. Nature 191, 368-370.

Upadhyaya, H.D., Gowda, C.L.L., Pundir, R.P.S., Reddy, G.V., Singh, S., 2006. Development of core sub set of finger millet germplasm using geographical origin and data on 14 quantitative traits. Genet. Resour. Crop Evol. 53, 679-685.

Upadhyaya, H.D., Gowda, C.L.L., Reddy, G.V., 2007a. Morphological diversity in finger millet germplasm introduced from southern and eastern Africa. J. SAT Agric. Res. 3, 1-3.

Upadhyaya, H.D., Pundir, R.P.S., Gowda, C.L.L., 2007b. Genetic resources diversity of finger millet-a global perspective. In: Mgonja, M.A., Lenne, J.M., Manyasa, E., Srinivasaprasad, S. (Eds.), Finger Millet Blast Management in East Africa. Creating Opportunities for Improving Production and Utilization of Finger Millet. Proceedings of the First International Finger Millet Stakeholder Workshop, Projects R8030 and R8445 UK Department for International Development-Crop Protection Programme, 13-14 September 2005, Nairobi. International Crops Research Institute for the Semi-Arid Tropics, Patancheru 502324, Andhra Pradesh, India pp. 90-101.

Underwood, R.A., 2000. Overcoming micronutrient deficiencies in developing countries: is there a role for agriculture? Food Nutr. Bull. 21, 356-360.

Wald, Abraham, 1943. Tests of statistical hypotheses concerning several parameters when the number of observations is large. Trans. Am. Math. Soc. 54, 426-482.

Ward, J.H., 1963. Hierarchical grouping to optimize an objective function. J. Am. Stat. Assoc. 58, 236-244.

Weissenberg, M., Levy, A., Schaeffler, I., Menagem, E., Barzilai, M., 1997. Rapid isocratic HPLC analysis of beta-carotene in red peppers (Capsicum annuum L.) and food preparations. Chromatographia 46, 399-403.

Welch, R.M., Graham, R.D., 2004. Breeding for micronutrients in staple food crops from a human nutrition perspective. J. Exp. Bot. 55, 353-364. 